\title{
Efficacy of caspofungin combined with trimethoprim/sulfamethoxazole as first-line therapy to treat non-HIV patients with severe pneumocystis pneumonia
}

\author{
GENSHENG ZHANG $^{1 *}$, MIAOMIAO CHEN ${ }^{1,2^{*}}$, SHUFANG ZHANG ${ }^{3}$, HONGWEI ZHOU $^{4}$, \\ XIAOZHEN JI ${ }^{5}$, JIACHANG CAI ${ }^{4}$, TIANZHENG LOU ${ }^{2}$, WEI CUI ${ }^{1}$ and NING ZHANG ${ }^{2}$ \\ ${ }^{1}$ Department of Critical Care Medicine, The Second Affiliated Hospital, Zhejiang University School of Medicine, \\ Hangzhou, Zhejiang 310009; ${ }^{2}$ Department of Critical Care Medicine, Lishui People's Hospital, Lishui, \\ Zhejiang 323000; ${ }^{3}$ Department of Cardiology; ${ }^{4}$ Microbiology Laboratory, The Second Affiliated Hospital, \\ Zhejiang University School of Medicine, Hangzhou, Zhejiang 310009; ${ }^{5}$ Department of Critical Care Medicine, \\ Longquan People's Hospital, Lishui, Zhejiang 323700, P.R. China
}

Received June 6, 2017; Accepted September 22, 2017

DOI: 10.3892/etm.2017.5516

\begin{abstract}
Combined treatment with caspofungin and trimethoprim/sulfamethoxazole (TMP/SMZ) as salvage therapy in non-HIV positive patients with severe pneumocystis pneumonia (PCP) yields poor outcomes. It remains unknown whether the use of this combination strategy as a first-line therapy would improve patient outcomes. The present study aimed to assess the efficacy of caspofungin combined with TMP/SMZ as a first-line therapy in non-HIV positive patients with severe PCP. A retrospective cohort study was conducted between March 2016 and February 2017. Patient clinical characteristics and outcomes were compared between two groups receiving first-line and second-line therapy respectively. In addition, similar cases from previous studies were assessed. A total of 14 patients were included in the present study (mean age, 58.79 \pm 14.41 years); including 9 patients receiving caspofungin and TMP/SMZ as a first-line therapy and 5 that received it as a second-line therapy. The overall positive response rate was $71.43 \%$ (10/14), with $88.89(8 / 9)$ and $40.00 \%(2 / 5)$ in the first-line and second-line therapy groups, respectively $(\mathrm{P}=0.095)$. The positive response rates of patients requiring invasive mechanical ventilation differed significantly between the first-line $(5 / 6,83.33 \%)$ and the second-line $(0 / 3,0 \%)$ therapy groups $(\mathrm{P}=0.048)$. All-cause
\end{abstract}

Correspondence to: Dr Gensheng Zhang, Department of Critical Care Medicine, The Second Affiliated Hospital, Zhejiang University School of Medicine, 88 Jiefang Road, Hangzhou, Zhejiang 310009, P.R. China

E-mail: genshengzhang@zju.edu.cn

*Contributed equally

Key words: pneumocystis pneumonia, caspofungin, trimethoprim/sulfamethoxazole, first-line therapy, second-line therapy hospital mortality was $42.86 \%(6 / 14)$, with mortality rates of $33.33(3 / 9)$ and $60.00 \%(3 / 5)$ in the first-line and second-line therapy groups, respectively $(\mathrm{P}=0.580)$. Combined with previously reported cases $(n=27)$, the positive response rate was significantly greater in the first-line therapy group $(11 / 12,91.67 \%)$ than in the second-line therapy group $(8 / 15$, $53.33 \%, \mathrm{P}=0.043)$. No significant differences were in all-cause mortality rates between the two groups (25.00 vs. $46.67 \%$, $\mathrm{P}=0.424$ ) were identified, despite the fact that all-course mortality in the first-line therapy group was $\sim 50 \%$ that of the second-line therapy group. Therefore, the results of the present study indicate that combined caspofungin and TMP/SMZ as first-line therapy may be a promising and effective strategy to treat non-HIV positive patients with severe PCP, particularly for those requiring invasive mechanical ventilation.

\section{Introduction}

Pneumocystis pneumonia (PCP) is a severe and life-threatening opportunistic infection that occurs in immunocompromised patients (1). The routine use of highly active antiretroviral therapy and PCP prophylaxis in patients with HIV has decreased the incidence and mortality rates of PCP $(1,2)$. However, the incidence of PCP in non-HIV positive patients has steadily increased due to the widespread use of immunosuppressive agents and corticosteroids (2). Despite the use of trimethoprim/sulfamethoxazole (TMP-SMZ) as first-line therapy, mortality rates in non-HIV positive patients diagnosed with PCP remain high. The overall mortality rate of patients with PCP is $48-67 \%(3,4)$ and is $84.2 \%$ in patients with severe PCP (5). Therefore, the treatment of non-HIV positive patients with severe PCP remains challenging and alternative therapeutic strategies are required.

Caspofungin is a novel antifungal agent that inhibits the synthesis of $\beta-1,3$-glucan in the cell wall (6). It may be an effective treatment for PCP, as $\beta$-1,3-glucan is the primary component of the cyst cell wall in Pneumocystis jirovecii (P. jirovecii) (7). TMP-SMZ affects trophic forms of $P$. jirovecii $(8,9)$, whereas 
caspofungin primarily acts on cyst forms of $P$. jirovecii (6); therefore, caspofungin may be used to treat severe or refractory PCP in combination with TMP/SMZ. In a mouse model of PCP, Lobo et al (10) demonstrated that the efficacy of low-dose caspofungin (0.001-0.1 mg/kg/day) combined with the standard-prophylactic dose of TMP/SMZ (12.5/62.5 mg/day), was higher than that of each drug alone $(\mathrm{P}<0.05)$. The synergistic effects of caspofungin and TMP/SMZ in the treatment of PCP have also been described in several case reports of non-HIV positive patients with severe $\mathrm{PCP}(8,9,11,12)$. However, in a case series including 4 non-HIV positive patients with severe PCP, no patients responded to combination treatment, which was used as second-line therapy following TMP/SMZ treatment failure (13). Therefore, it remains unclear whether combination treatment with TMP/SMZ and caspofungin as first-line therapy is more effective than its use as second-line therapy to treat severe PCP.

Therefore, the present retrospective cohort study aimed to comparatively assess caspofungin and TMP/SMZ combination as first and second-line therapies to treat non-HIV positive patients with severe PCP. Data from cases included in previous studies were included in the analysis.

\section{Patients and methods}

Patients. The present study was performed at the Second Affiliated Hospital, Zhejiang University School of Medicine (Hangzhou, China), a 3,200-bed tertiary care teaching hospital in China. Consecutive cases, assessed by polymerase chain reaction (PCR) and/or Grocott's methenamine silver (GMS) staining of bronchoalveolar lavage fluid (BALF), sputum, or trachea aspirate samples taken from patients with suspected PCP, were identified by screening the laboratory database. A total of 65 patients with suspected PCP were recruited to the present study from March 2016 to February 2017. The mean age of the patients was $59.36 \pm 11.56$ years and $47.69 \%(31 / 65)$ of them were males.

The present study received human research ethics approval (no. 2017-022) from the Ethics Committee of The Second Affiliated Hospital, School of Medicine, Zhejiang University. Due to the retrospective nature of the study, the Ethics Committee determined that no patient consent was required. In addition, as the manuscript did not include any personal information, a statement of permission from patients for submission to the present study was not required.

Microbiological methods. Bronchoalveolar lavage was performed through a fiber-optic bronchoscope using standard techniques. Following local anesthesia of the airways with 2\% lidocaine spray (Xiangxue Pharmaceutical Co., Ltd., Guangzhou, China), a fiber-optic bronchoscope was inserted into the lung and inserted into a peripheral airway of the lingula, right middle lobe or lesion localized lobe. Sterile saline (5x20 ml aliquots) at $37^{\circ} \mathrm{C}$ was instilled through the bronchoscope and the fluid was immediately removed via suction with $50-100 \mathrm{mmHg}$ of negative pressure. A total of 40-60\% of the infused volume was recovered (14). BALF, sputum and trachea aspirate were extracted for GMS staining and PCR for PCP when PCP was suspected following hospitalization.
GMS staining was performed following a routine laboratory protocol. Briefly, samples of BALF, sputum, and trachea aspirate were centrifuged at $603.7 \mathrm{x} \mathrm{g}$ for $5 \mathrm{~min}$ at room temperature and the sediment was smeared. Slides were then stained with periodate solution for 10-15 $\mathrm{min}$ at room temperature, fresh hexamine silver solution (BA4094; BaSO Biotechnology Co., Ltd., Wuhan, China) at $62^{\circ} \mathrm{C}$ for $30 \mathrm{~min}$, sodium thiosulfate solution for $3 \mathrm{~min}$ at room temperature and hematoxylin-eosin staining solution for 30-60 sec at room temperature, respectively. Slides were then examined using a light microscope (Fig. 1).

PCR was performed using specific primers to amplify the mitochondrial large subunit rRNA (mtLSUrRNA) gene of $P$. jirovecii. Total DNA from each sample was extracted using the Axygen Bacterial Genome Extraction kit (Axygen; Corning Inc., Corning, NY, USA) following the manufacturer's instructions. PCR amplification primers were synthesized by Sangon Biotech Co., Ltd. (Shanghai, China). The sequence of the primers were as follows: pAZ102-E sense, 5'-GATGGC TGTTTCCAAGCCCA-3' and pAZ102-H antisense, 5'-GTG TACGTTGCAAAGTACTC-3', and a $20 \mu \mathrm{l}$ reaction system including Taq DNA polymerase (Takara Biotechnology Co., Ltd., Dalian, China) was used. The amplification fragment was $346 \mathrm{bp}$ long and the PCR amplification was performed including initially denaturing at $94^{\circ} \mathrm{C}$ for $5 \mathrm{~min}$ followed by amplification for 30 cycles and a final extension. Each cycle consisted of denaturation at $94^{\circ} \mathrm{C}$ for $30 \mathrm{sec}$, followed by annealing at $52^{\circ} \mathrm{C}$ for $30 \mathrm{sec}$, then elongation at $72^{\circ} \mathrm{C}$ for $30 \mathrm{sec}$, followed by a final extension at $72^{\circ} \mathrm{C}$ for $5 \mathrm{~min}$. PCR products were detected by $1 \%$ Agar agarose gel electrophoresis and visualized with ultraviolet light. PCR amplification fragments with the bands of the correct length (346 bp) were sent to Sangon Biotech Co., Ltd., for sequencing. Sequences were compared with those listed in the GenBank database (http://blast.ncbi.nlm.nih.gov/Blast.cgi; Fig. 2), and PCR amplification fragments with correct sequence were positive.

Inclusion and exclusion criteria. The inclusion criteria were as follows: i) The presence of relevant pulmonary symptoms, including fever, cough or dyspnea; ii) pulmonary infiltration indicated by chest radiography or computed tomography (CT), which was performed when PCP was clinically suspected following hospitalization; iii) microbiological confirmation by positive PCR and/or GMS results for sputum, aspirate or BALF samples; iv) severe PCP; and v) treatment with caspofungin combined with TMP/SMZ. Patients that were $<18$ years old, HIV positive or pregnant were excluded from the present study.

Data collection. Clinical data were retrieved from medical records. These included data regarding demographic characteristics; underlying diseases; immunosuppressive conditions in the 3 months preceding PCP onset; clinical symptoms and laboratory test results on admission; radiological images; illness severity parameters, including oxygenation index $\left(\mathrm{PaO}_{2} / \mathrm{FiO}_{2}\right)$, and acute physiology and chronic health evaluation (APACHE) II score on the day of acute respiratory failure (ARF); the time of PCP symptom onset; duration of PCP treatment and the PCP treatment regimen administered. The PCP treatment regimen administered in the present study was 


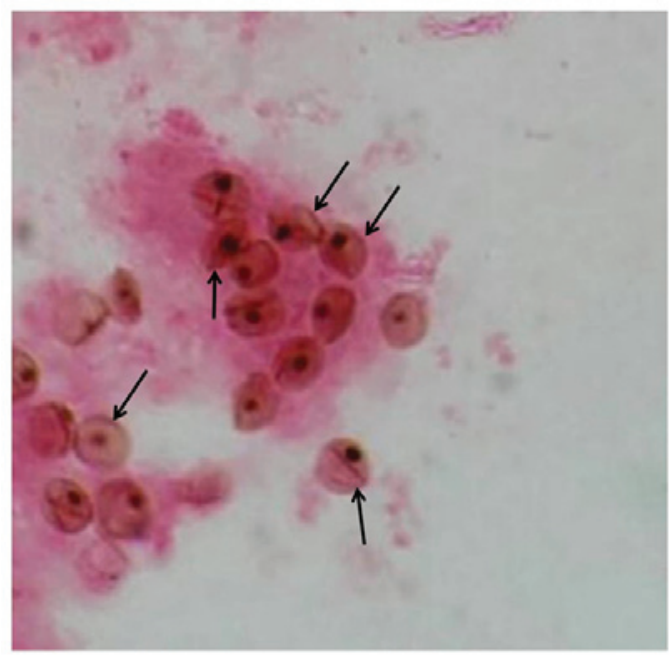

Figure 1. Cysts of Pneumocystis jirovecii grown from bronchoalveolar lavage fluid (indicated by black arrows) (Grocott's methenamine silver staining; MS and eosin, magnification, $\mathrm{x} 1,000)$.

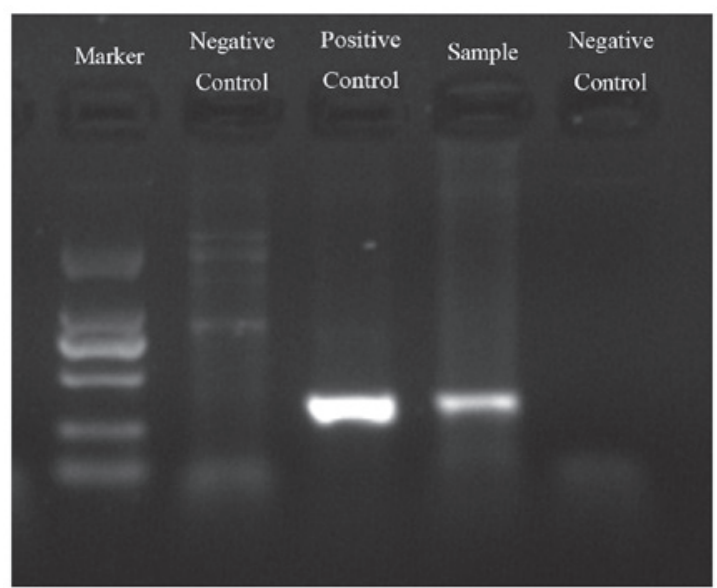

Figure 2. Agarose gel electrophoresis indicating typical polymerase chain reaction amplification findings of the mitochondrial large subunit ribosomal RNA gene of Pneumocystis jirovecii.

a combination of TMP/SMZ and caspofungin, used as a firstor second-line therapy. The primary endpoint was all-cause in hospital mortality and the secondary endpoint was the positive response rate to the PCP treatment.

Systematic review of literature. The PubMed database (www.ncbi.nlm.nih.gov/pmc/) was screened on December 31, 2016 using the key words 'Pneumocystis pneumonia', 'Pneumocystis jirovecii pneumonia', 'Pneumocystis carinii pneumonia' and 'caspofungin'. Studies were only included if they were written in English and evaluated PCP patients treated with caspofungin combined with TMP/SMZ . Titles, abstracts, and full texts of all relevant articles were subsequently screened for those which included patients with severe PCP diagnosed based on pulmonary symptoms, pulmonary imaging expressions and positive PCR, and/or GMS results for respiratory secretion samples simultaneously. Studies that had included patients that were $<18$ years, HIV positive or pregnant were excluded. The following parameters from previous studies were analyzed: Demographic characteristics, underlying diseases, co-infection, PCP treatment regimens, response to PCP treatment and all-cause in-hospital mortality.

Definitions. The classification of PCP severity was based on partial arterial oxygen pressure $\left(\mathrm{PaO}_{2}\right)$ while breathing room air or the alveolar-arterial oxygen difference $\left(\mathrm{A}-\mathrm{aDO}_{2}\right)$, as follows: Mild, $\mathrm{PaO}_{2}>70 \mathrm{mmHg}$ or A-aDO2 $<35 \mathrm{mmHg}$; moderate, $\mathrm{PaO}_{2} \leq 70 \mathrm{mmHg}$ or $\mathrm{A}-\mathrm{aDO}_{2} \geq 35 \mathrm{mmHg}$; severe, $\mathrm{PaO}_{2}<60 \mathrm{mmHg}$ or A-aDO $2 \geq 45 \mathrm{mmHg}(15,16) . \mathrm{PaO}_{2}$ was measured directly by blood gas analysis of arterial blood and A-aDO $\mathrm{aDas}_{2}$ calculated via the following formula: $\mathrm{A}-\mathrm{aDO}_{2}=$ $\mathrm{FiO}_{2} \times 713-\mathrm{PaCO}_{2} / \mathrm{R}-\mathrm{PaO}_{2}$; where $\mathrm{FiO}_{2}=$ fraction of inspiration oxygen, $\mathrm{PaCO}_{2}=$ partial arterial carbon dioxide pressure and $\mathrm{R}=$ respiratory quotient. The above parameters were obtained via blood gas analysis of arterial blood. In the present study, first-line therapy was defined as the initial PCP treatment administered and second-line therapy was defined as the regimen administered following failed monotherapy with caspofungin or TMP/SMZ. Treatment failure was defined as the worsening of hypoxia or radiographical features 4 days after first-line therapy (15). Positive response to PCP treatment was defined as: i) Amelioration or resolution of baseline signs, symptoms and chest roentgenographic findings; and ii) decreased oxygen requirement following therapy $(13,17)$.

Statistical analysis. Continuous variables were presented as the mean \pm standard deviation if normally distributed. Skewed data were presented as the median and interquartile ranges. For small sample sizes, all continuous variables were analyzed using the non-parametric Mann-Whitney U test. Categorical variables were expressed as percentages and compared using Fisher's exact test. All statistical analyses were performed using the SPSS statistical software (version 20; IBM, Inc., Armonk, NY, USA). $\mathrm{P}<0.05$ was considered to indicate a statistically significant difference.

\section{Results}

Patient characteristics. In the present study, 65 patients with suspected PCP were assessed by PCR and/or Grocott's methenamine silver staining of BALF, trachea aspirate or sputum samples. Among them, 51 subjects were excluded; therefore, 14 non-HIV positive patients with severe PCP were included in the present study (Fig. 3). Included patients had a mean age of $58.79 \pm 14.41$ years and the proportion of male patients was $28.57 \%$ (4/14). The most common underlying conditions were autoimmune diseases $(35.71 \%, 5 / 14)$, hematological diseases $(21.43 \%, 3 / 14)$ and solid tumors $(14.29 \%, 2 / 14)$. All patients had existing immunosuppressive conditions in the 3 months preceding PCP onset and the majority received medication with corticosteroids and/or immunosuppressants $(71.43 \%$, 10/14; Table I). The patients were divided into 2 groups. One group consisted of 9 patients that received caspofungin and TMP/SMZ as a first-line therapy. The other group consisted of 5 patients that received the same treatment as a second-line therapy. There were no significant differences in age, sex, underlying diseases and immunosuppressive conditions, between the first-line and second-line therapy 
Table I. Baseline characteristics of the subjects.

\begin{tabular}{|c|c|c|c|c|}
\hline Variables & Total $(\mathrm{n}=14)$ & First-line $^{c}(n=9)$ & Second-line ${ }^{c}(n=5)$ & P-value \\
\hline Age, mean \pm SD & $58.79 \pm 14.41$ & $57.67 \pm 13.46$ & $60.80 \pm 17.46$ & 0.606 \\
\hline Male, n (\%) & $4(28.57)$ & $3(33.33)$ & $1(20.00)$ & 1.000 \\
\hline \multicolumn{5}{|l|}{ Underlying diseases $(\mathrm{n}, \%)$} \\
\hline Autoimmune diseases & $5(35.71)$ & $2(22.22)$ & $3(60.00)$ & \multirow[t]{6}{*}{0.940} \\
\hline Hematological diseases & $3(21.43)$ & $2(22.22)$ & $1(20.00)$ & \\
\hline Chronic kidney diseases & $2(14.29)$ & $1(11.11)$ & $1(20.0)$ & \\
\hline Solid tumor & $2(14.29)$ & $2(22.22)$ & $0(0)$ & \\
\hline Solid tumor ${ }^{\mathrm{a}}$ & $1(7.14)$ & $1(11.11)$ & $0(0)$ & \\
\hline Diabetes mellitus & $1(7.14)$ & $1(11.11)$ & $0(0)$ & \\
\hline \multicolumn{5}{|l|}{ Immunosuppressions (n, \%) } \\
\hline Corticosteriod & $6(42.86)$ & $4(44.44)$ & $2(40.01)$ & \multirow[t]{5}{*}{0.409} \\
\hline Immunosuppressants ${ }^{\mathrm{b}}$ & $4(28.57)$ & $1(11.11)$ & $3(60.01)$ & \\
\hline Chemotherapy & $1(7.14)$ & $1(11.11)$ & $0(0)$ & \\
\hline Chemoradiotherapy & $2(14.29)$ & $2(22.22)$ & $0(0)$ & \\
\hline Radiotherapy and corticosteriod & $1(7.14)$ & $1(11.11)$ & $0(0)$ & \\
\hline
\end{tabular}

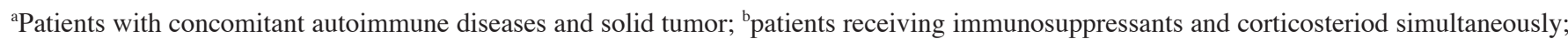
cpatients receiving caspofungin and TMP/SMZ as first-line therapy or as second-line therapy. SD, standard deviation; TMP/SMZ, trimethoprim/sulfamethoxazole.

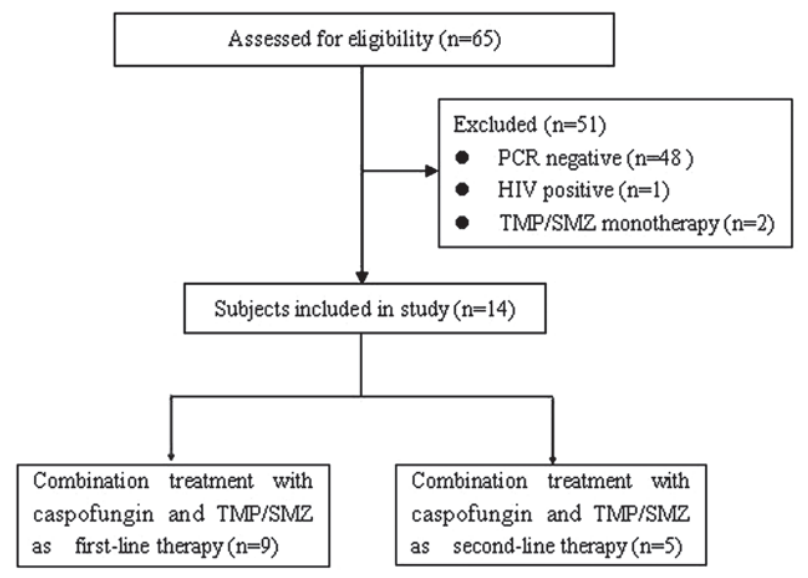

Figure 3. Flowchart of recruited patients.

groups, indicating that the baseline characteristics of the two groups were similar.

Clinical manifestations and assisted examination. The clinical symptoms of PCP on admission included fever $(100.00 \%, 14 / 14)$, dyspnea $(92.86 \%, 13 / 14)$ and cough $(64.29 \%, 9 / 14)$. All patients underwent chest CT scans; the most common finding was bilateral diffuse ground glass opacity (GGO; 100.00\%, 14/14), followed by bilateral predominant consolidation $(28.57 \%, 4 / 14$; Table II). All patients experienced acute respiratory failure (ARF), with $64.29 \%(9 / 14)$ requiring invasive mechanical ventilation (Table III). $\mathrm{PaO}_{2} / \mathrm{FiO}_{2}$ values and APACHE II scores on the day of ARF were $146.56 \pm 63.51 \mathrm{mmHg}$ and $16.64 \pm 5.42$, respectively (Table II). A total of 5 patients had co-infections, including bacterial pneumonia $(21.43 \%, 3 / 14)$, bacteremia $(7.14 \%, 1 / 14)$ and spontaneous ascitic fluid infection $(7.14 \%$, $1 / 14)$, and 1 patient experienced bacterial pneumonia and pulmonary aspergillosis simultaneously $(7.14 \%, 1 / 14$; Table II). These parameters, including clinical symptoms and assisted examination date, as well as the co-infections and illness severity indices including $\mathrm{PaO}_{2} / \mathrm{FiO}_{2}$ and APACHE II scores, were similar between the first-line and second-line therapy groups (all P>0.05; Table II).

PCP treatment and outcomes. No patients underwent prophylactic treatment prior to $\mathrm{PCP}$ onset, or received sulfa or caspofungin for other diseases prior to the initiation of PCP treatment. All patients were treated with adjunctive corticosteroids. In the present study, patients received $70 \mathrm{mg}$ caspofungin intravenously on the first day. Caspofungin was subsequently administered at a dose of $50 \mathrm{mg} /$ day. TMP/SMZ was orally administered at a mean dose of $14.71 \pm 2.39 \mathrm{mg} / \mathrm{kg} / \mathrm{day}$. The duration of treatment with caspofungin and TMP/SMZ was $21.88 \pm 6.53$ days, in the first-line therapy group; whereas the duration of treatment with caspofungin was $13.00 \pm 5.65$ days and with TMP/SMZ was $16.00 \pm 5.66$ days in the first-line therapy group. The time from symptom onset to PCP treatment was 12.64 \pm 8.79 days, with no statistically significant difference between the two groups $(11.89 \pm 8.55$ vs. $14.00 \pm 10.07$ days; $\mathrm{P}=0.797)$. The first-line therapy group received caspofungin with TMP/SMZ 8 days earlier than the second-line therapy group $(11.89 \pm 8.55$ vs. $20.20 \pm 10.07 ; \mathrm{P}=0.190)$. The duration of $\mathrm{PCP}$ treatment and length of hospital stay were $20.70 \pm 6.55$ and $34.90 \pm 15.55$ days, respectively. These did not differ significantly between the two treatment groups (Table III). The overall positive response rate to PCP treatment was $71.43 \%(10 / 14)$ and was 
Table II. Clinical manifestations and auxiliary examination of patients.

\begin{tabular}{|c|c|c|c|c|}
\hline Variables & Total $(n=14)$ & First-line $^{a}(n=9)$ & Second-line $^{a}(n=5)$ & P-value \\
\hline \multicolumn{5}{|l|}{ Symptom (n, \%) } \\
\hline Fever & $14(100.00)$ & $6(100.00)$ & $4(100.00)$ & 1.000 \\
\hline Cough & $9(64.29)$ & $6(66.67)$ & $3(60.00)$ & 1.000 \\
\hline Dyspnea & $13(92.86)$ & $9(100.00)$ & $4(80.00)$ & 0.357 \\
\hline \multicolumn{5}{|l|}{ Severity on the day of ARF } \\
\hline $\mathrm{PaO}_{2} / \mathrm{FiO}_{2}$ & $146.56 \pm 63.51$ & $149.59 \pm 75.05$ & $141.11 \pm 42.25$ & 0.797 \\
\hline APACHE II score & $16.64 \pm 5.42$ & $15.78 \pm 4.82$ & $18.20 \pm 6.65$ & 0.438 \\
\hline \multicolumn{5}{|l|}{ Laboratory results } \\
\hline White blood cell count, cells/ml & $7.04 \pm 2.67$ & $7.53 \pm 2.74$ & $6.16 \pm 2.56$ & 0.518 \\
\hline Lymphocyte counts, cells/ml, median counts, IQR & $0.56(0.36-0.67)$ & $0.59(0.33-0.89)$ & $0.53(0.34-0.70)$ & 1.000 \\
\hline Lactate dehydrogenase, $\mathrm{U} / \mathrm{l}$ & $593.47 \pm 212.88$ & $626.10 \pm 234.86$ & $534.74 \pm 173.95$ & 0.699 \\
\hline Serum $\beta-1,3$-glucan ${ }^{\mathrm{b}}, \mathrm{pg} / \mathrm{ml}(\mathrm{n}=11)$ & $513.06 \pm 289.65$ & $472.06 \pm 281.41$ & $584.83 \pm 332.39$ & 0.412 \\
\hline \multicolumn{5}{|l|}{ Radiographic findings (n, \%) } \\
\hline Bilateral GGOs & $14(100.00)$ & $9(100.00)$ & $5(100.00)$ & 1.000 \\
\hline Consolidation & $4(28.57)$ & $3(33.33)$ & $1(20.00)$ & 1.000 \\
\hline \multicolumn{5}{|l|}{ Co-infections (n, \%) } \\
\hline Bacterial pneumonia & $3(21.43)$ & $2(22.22)$ & $1(20.00)$ & 0.895 \\
\hline Pulmonary aspergillosis ${ }^{c}$ & $1(7.14)$ & $1(11.11)$ & $0(0)$ & \\
\hline Bacteremia & $1(7.14)$ & $1(11.11)$ & $0(0)$ & \\
\hline Spontaneous ascitic fluid infection & $1(7.14)$ & $0(0)$ & $1(20.00)$ & \\
\hline
\end{tabular}

${ }^{a}$ Patients receiving caspofungin and TMP/SMZ as first- or second-line therapy; ${ }^{\mathrm{b}} \beta$-1,3-glucan levels of three patients were not measured; cpatient experienced bacterial pneumonia and pulmonary aspergillosis simultaneously. IQR, interquartile range; GGO, ground glass opacitie; ARF, acute respiratory failure; APACHE II, acute physiology and chronic health evaluation; TMP/SMZ, trimethoprim/sulfamethoxazole; ICU, intensive care unit.

$88.89 \%(8 / 9)$ and $40.00 \%(2 / 5)$ in the first-line and second-line therapy groups, respectively $(\mathrm{P}=0.095)$. Similarly, all-cause in-hospital mortality in the present study was $42.86 \%(6 / 14)$, with $33.33 \%(3 / 9)$ and $60.00 \%(3 / 5)$ in the first-line and second-line therapy groups, respectively ( $\mathrm{P}=0.580$; Table III). The efficacies of both combination treatments in patients requiring invasive mechanical ventilation were further analyzed. A total of 9 patients $(9 / 14,64.29 \%)$ were supported by mechanical ventilation, including $6(66.67 \%, 6 / 9)$ and 3 $(60.00 \%, 3 / 5)$ that received caspofungin with $\mathrm{TMP} / \mathrm{SMZ}$ as first-line and second-line therapies, respectively. In this sub-population, the positive response rate to caspofungin with TMP/SMZ as a first-line therapy was significantly higher than that of the group receiving it as a second-line therapy (83.33 vs. $0 \%$; $\mathrm{P}=0.048$ ). All-cause mortality in patients that received caspofungin with TMP/SMZ as a first-line therapy was half that of the second-line therapy group, although this difference was not significant (50 vs. $100 \%$; $\mathrm{P}=0.464$; Table III).

Analysis including previously reported cases. To further explore the role of the aforementioned treatment as a first-line therapy, 13 non-HIV positive patients with severe PCP treated with the combination of caspofungin and TMP/SMZ were selected from previously reported cases $(8,9,11-13)$. Among them, 3 patients received caspofungin and TMP/SMZ as a first-line therapy $(8,9,12)$ and the remaining 10 received it as a second-line therapy $(8,9,11,13)$. There were 27 patients recruited from the present study and previous reports $(8,9,11-13)$; patient characteristics and treatment outcomes are summarized in Table IV. The 27 patients had a mean age of $52.28 \pm 19.11$ years and included (15/27) 55.56\% males. The primary underlying conditions observed in patients were solid organ transplants $(37.04 \%$, 10/27), hematological diseases $(22.22 \%, 6 / 27)$ and autoimmune diseases $(18.52 \%, 5 / 27)$. A total of 16 patients $(59.26 \%)$ had co-infections, including 5 with bacterial pneumonia $(18.52 \%$, 5/27), 3 with viral pneumonia $(11.11 \%, 3 / 27)$ and 6 with extrapulmonary infection $(22.22 \%, 6 / 27)$. The duration of PCP treatment was $19.88 \pm 7.08$ days; the overall positive response rates to the PCP treatment and all-cause in-hospital mortality rates were $70.37 \%(19 / 27)$ and $37.04 \%$ (10/27), respectively. Age, sex, underlying diseases, co-infections and treatment course were similar between the two groups. However, the positive response rate to PCP treatment was significantly higher in patients treated with combined caspofungin and TMP/SMZ as a first-line therapy than in those administered the same treatment as a second-line therapy (91.67 vs. 53.33\%; $\mathrm{P}=0.043$ ). Although no significant differences were identified, the combined treatment as a first-line therapy reduced the all-course mortality rate to a greater extent than when it was used as a second-line therapy (25.00 vs. $46.67 \%$, respectively; $\mathrm{P}=0.424$; Table IV). 
Table III. PCP treatment and patient outcomes.

\begin{tabular}{|c|c|c|c|c|}
\hline Variables & Total $(n=14)$ & First-line $^{\mathrm{a}}(\mathrm{n}=9)$ & Second-line $^{\mathrm{a}}(\mathrm{n}=5)$ & P-value \\
\hline \multicolumn{5}{|l|}{ Interval } \\
\hline Symptom onset to admission & $9.86 \pm 6.67$ & $8.33 \pm 5.81$ & $12.60 \pm 7.89$ & 0.379 \\
\hline Admission to PCP treatment, median days (IQR) & $0(0-7.00)$ & $0(0-8.00)$ & $0(0-3.50)$ & 0.350 \\
\hline Symptom onset to PCP treatment (d) & $12.64 \pm 8.79$ & $11.89 \pm 8.55$ & $14.00 \pm 10.07$ & 0.797 \\
\hline Symptom onset to combination treatment (d) & $14.86 \pm 10.00$ & $11.89 \pm 8.55$ & $20.20 \pm 10.07$ & 0.190 \\
\hline Dose of TMP/SMZ ${ }^{\mathrm{b}}, \mathrm{mg} / \mathrm{kg} /$ day & $14.71 \pm 2.39$ & $15.05 \pm 2.21$ & $14.10 \pm 2.85$ & 0.518 \\
\hline Treatment duration, $\mathrm{d}(\mathrm{n}=8)$ & $20.70 \pm 6.55$ & $21.88 \pm 6.53$ & $16.00 \pm 5.66$ & 0.400 \\
\hline Admission to ICU, n (\%) & $9(64.29)$ & $6(66.67)$ & $3(60.00)$ & 1.000 \\
\hline Length of hospital stay $(d)(n=8)$ & $34.90 \pm 15.55$ & $36.00 \pm 17.44$ & $30.50 \pm 0.71$ & 0.711 \\
\hline Positive response rate, $\mathrm{n}(\%)$ & $10(71.43)$ & $8(88.89)$ & $2(40.00)$ & 0.095 \\
\hline All-cause hospital mortality, n (\%) & $6(42.86)$ & $3(33.33)$ & $3(60.00)$ & 0.580 \\
\hline Invasive mechanical ventilation, n (\%) & $9(64.29)$ & $6(66.67)$ & $3(60.00)$ & 1.000 \\
\hline Positive response rate, n (\%) & $5(55.56)$ & $5(83.33)$ & $0(0)$ & 0.048 \\
\hline All-cause hospital mortality, n (\%) & $6(66.67)$ & $3(50.00)$ & $3(100.0)$ & 0.464 \\
\hline
\end{tabular}

aPatients receiving caspofungin and TMP/SMZ as first- or second-line therapy; ${ }^{b}$ dose of TMP/SMZ was based on TMP; IQR, interquartile range; TMP/SMZ, trimethoprim/sulfamethoxazole; PCP, pneumocystis pneumonia; ICU, intensive care unit.

Table IV. Clinical characteristics and outcomes of pooled cases.

\begin{tabular}{|c|c|c|c|c|}
\hline Variables & Total $(n=27)$ & First-line $^{a}(\mathrm{n}=12)$ & Second-line $^{a}(\mathrm{n}=15)$ & P-value \\
\hline Age, mean $\pm \mathrm{SD}$ & $52.28 \pm 19.11$ & $52.08 \pm 19.22$ & $52.43 \pm 20.76$ & 0.905 \\
\hline Male, n (\%) & $15(55.56)$ & $5(41.67)$ & $10(66.67)$ & 0.258 \\
\hline \multicolumn{5}{|l|}{ Underlying diseases, n (\%) } \\
\hline Solid organ transplant & $10(37.04)$ & $2(16.7)$ & $8(53.33)$ & \multirow[t]{7}{*}{0.245} \\
\hline Hematological diseases & $6(22.22)$ & $3(25.0)$ & $3(20.00)$ & \\
\hline Autoimmune diseases & $5(18.52)$ & $2(16.67)$ & $3(20.00)$ & \\
\hline Solid tumor & $2(7.41)$ & $2(16.67)$ & $0(0)$ & \\
\hline Solid tumor ${ }^{\mathrm{b}}$ & $1(3.70)$ & $1(8.33)$ & $0(0)$ & \\
\hline Chronic kidney diseases & $2(7.41)$ & $1(8.33)$ & $1(6.7)$ & \\
\hline diabetes mellitus & $1(3.70)$ & $1(8.33)$ & $0(0)$ & \\
\hline \multicolumn{4}{|l|}{ Co-infections, n (\%) } & \multirow[t]{6}{*}{0.502} \\
\hline Bacterial pneumonia & $5(18.52)$ & $3(25.00)$ & $2(13.33)$ & \\
\hline Viral pneumonia $^{c}$ & $3(11.11)$ & $0(0)$ & $3(20.0)$ & \\
\hline Pulmonary nocardiosis & $1(3.70)$ & $0(0)$ & $1(6.67)$ & \\
\hline Pulmonary aspergillosis ${ }^{c}$ & $1(3.70)$ & $1(8.33)$ & $0(0)$ & \\
\hline Extrapulmonary infection & $6(22.22)$ & $3(25.00)$ & $3(20.00)$ & \\
\hline Treatment duration, $\mathrm{d}$ & $19.88 \pm 7.08$ & $19.00 \pm 7.89$ & $20.86 \pm 6.42$ & 0.606 \\
\hline Positive response rate, $\mathrm{n}(\%)$ & $19(70.37)$ & $11(91.67)$ & $8(53.33)$ & 0.043 \\
\hline The all-cause hospital mortality, n (\%) & $10(37.04)$ & $3(25.00)$ & $7(46.67)$ & 0.424 \\
\hline
\end{tabular}

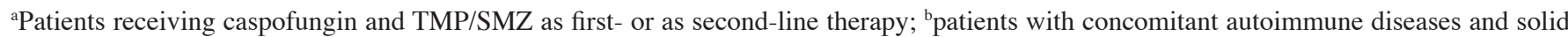
tumor; ${ }^{c}$ co-infected with bacterial pneumonia. $\mathrm{P}<0.05$ indicated a significant difference. SD, standard deviation; TMP/SMZ, trimethoprim/sulfamethoxazole.

\section{Discussion}

The present study demonstrated that all-cause in-hospital mortality in non-HIV positive patients with severe PCP treated with combined caspofungin and TMP/SMZ was $37.04 \%$. In the group of patients receiving caspofungin and TMP/SMZ as a first-line therapy, the in-hospital mortality rate was lower than in the second-line treatment group 
(25.00 vs. $46.67 \%)$. In previous studies, the mortality rate of non-HIV positive patients with severe PCP administered TMP/SMZ as a first-line therapy was $62-84.2 \%(4,5,18,19)$. The results of the present study suggest that caspofungin and TMP/SMZ exhibit synergistic effects in severe PCP, in accordance with the results of a previous study (10). The associated synergistic mechanisms may be as follows: Caspofungin may affect cyst formation by inhibiting $\beta$-glucan synthesis, whilst only exhibiting weak effects on trophic forms (6); similarly, TMP/SMZ may only affect trophic forms by interfering with folate metabolism $(8,9)$. Therefore, caspofungin and TMP/SMZ used in combination may inhibit the entire life cycle of Pneumocystis jirovecii. Alternatively, caspofungin may decrease $\beta$-glucan induced excessive inflammatory responses by inhibiting $\beta$-glucan synthesis (20), thus alleviating PCP. Finally, TMP/SMZ acts slowly, requiring 5-8 days to induce a curative effect (1), whereas caspofungin has a rapid onset, therefore it may be inferred that combinational treatment with TMP/SMZ and caspofungin may compensate the TMP/SMZ deficiency that occurs in patients receiving delayed PCP treatment. Although the results of the present study and those of a previous in vivo experiment (10) suggest that combination treatment with caspofungin and TMP/SMZ may be a promising strategy of treating severe PCP in patients without HIV, few studies have directly assessed the effects of this combination treatment on severe PCP among non-HIV patients compared with TMP/SMZ monotherapy; thus further prospective studies are required to confirm these results.

To the best of our knowledge, the present retrospective cohort study is the first to determine the efficacy of combination treatment with caspofungin and TMP/SMZ as a first-line therapy compared with as a second-line therapy to treat non-HIV positive patients with severe PCP. The positive response rate of the combination strategy increased from 40 when used as a second-line therapy to $88.9 \%$ when used as a first-line therapy. Additionally, all-course mortality rates from patients receiving combination therapy decreased by $\sim 50 \%$ when it was used as a first-line therapy compared with when it was used as a second-line therapy (33.33\% vs. 60.00, respectively). The improvements in positive responses and all-course mortality rates were unrelated to factors that are regularly attributed to the prognosis of PCP (21-25), as many were similar between the two groups. These included: Immunosuppressive conditions, lymphocyte count, radiological manifestations, the need for invasive mechanical ventilation and transfer to the intensive care unit, $\mathrm{PaO}_{2} / \mathrm{FiO}_{2}$, APACE II score, and co-infection.

Despite improvements in the positive response and all-cause mortality rates when the combined treatment was administered to patients as a first-line treatment as opposed to second-line therapy, no significant differences were identified. This may be due to the small number of patients included in the present study. Combined with data from previous studies $(8,9,11-13)$, the total sample size was increased to 27. The results of these pooled cases indicated that administration of caspofungin and TMP/SMZ combination treatment as first-line therapy significantly increased the positive response rate of patients from $53.33 \%$ in the second-line therapy group to $91.67 \%$ in the first-line therapy group.
The lack of a significant difference between the positive response and all-cause hospital mortality rates may be due to the variation in PCP progression among patients. Significantly higher positive response rates following treatment with caspofungin and TMP/SMZ as a first-line therapy were only observed in patients requiring invasive mechanical ventilation, who experienced clinical aggravation. Correspondingly, no patients that required invasive mechanical ventilation support in the second-line therapy group survived following treatment administration of the combined treatment, whereas $50 \%$ of patients in the first-line therapy group survived. These results indicate that it is necessary for patients requiring mechanical ventilation to receive caspofungin with TMP/SMZ as a first-line therapy; the combined therapeutic strategy may also be more effective in patients with severe PCP if administered as early as possible.

Finally, the mean duration from symptom onset to PCP treatment in the first-line therapy group included in the present study was 11.89 days, which may explain the lack of an identifiable significant difference in the positive response and all-cause hospital mortality rates between the two groups. This is important, as the time from symptom onset to diagnosis and treatment is a major risk factor for PCP mortality (1). Several factors may have contributed to this delay in treatment. PCP progression in non-HIV patients is relatively fast and peaks 3-6 days following onset, depending on disease severity (1). This may explain the average 8.33-day delay from symptom onset to hospital admission in the first-line therapy group observed in the present study, which may have been detrimental to the patients. The time period between hospital admission and PCP treatment may have also contributed to the delay in first-line therapy administration. The time between admission and PCP treatment in the first-line group included in the present study ranged from 0-8 days. Initiating PCP therapy within 7 days following symptom onset is important as intubation and mechanical ventilation may be avoided if treatment is prompt (1). Thus, PCP therapy initiation may have been too late for the non-HIV positive patients with severe PCP included in the present study. Taken together, the results indicate that combined treatment with caspofungin and TMP/SMZ as a first-line therapy may achieve better outcomes if it is administered to patients as soon as possible.

The present study had several limitations. Firstly, a positive PCR of $P$. jirovecii was an important diagnosis criterion for PCP in the present study, whereas it may also reflect a colonization of $P$. jirovecii. However, the included patients with PCP in our study had simultaneously relevant pulmonary symptoms and compatible radiologic findings besides microbiological confirmation by positive PCR and/or GMS results. Therefore, possibility that the included patients had only a colonization of $P$. jirovecii, without infection, was excluded. Furthermore, due to a small sample size, the present study may not have been able to accurately identify differences between the two treatment groups. To overcome these limitations and obtain more evidence, larger prospective randomized trials are necessary.

In conclusion, the clinical efficacy of caspofungin combined with TMP/SMZ as a first-line therapy is superior to its use as a second-line therapy to treat non-HIV positive patients with severe PCP, particularly in those requiring 
invasive mechanical ventilation. Therefore, the combination of caspofungin and TMP/SMZ as a first-line therapy may be a promising treatment strategy to treat non-HIV positive patients with severe PCP.

\section{Acknowledgements}

The present study was supported, in part, by grants from the National Natural Science Foundation of China (grant no. 81570017, G.S. Zhang). The authors wish to thank Dr Zexin Chen from the Department of Clinical Epidemiology and Biostatistics, The Second Affiliated Hospital, Zhejiang University School of Medicine, for help with statistical analysis.

\section{References}

1. Roux A, Gonzalez F, Roux M, Mehrad M, Menotti J, Zahar JR, Tadros VX, Azoulay E, Brillet PY and Vincent F; Groupe de recherche respiratoire en réanimation en onco-hématologie (Grrr-OH): Update on pulmonary Pneumocystis jirovecii infection in non-HIV patients. Med Mal Infect 44: 185-198, 2014.

2. Carmona EM and Limper AH: Update on the diagnosis and treatment of pneumocystis pneumonia. Ther Adv Respir Dis 5: 41-59, 2011

3. Festic E, Gajic O, Limper AH and Aksamit TR: Acute respiratory failure due to pneumocystis pneumonia in patients without human immunodeficiency virus infection: Outcome and associated features. Chest 128: 573-579, 2005.

4. Monnet X, Vidal-Petiot E, Osman D, Hamzaoui O, Durrbach A, Goujard C, Miceli C, Bouree P and Richard C: Critical care management and outcome of severe pneumocystis pneumonia in patients with and without HIV infection. Crit Care 12: R28, 2008.

5. Roembke F, Heinzow HS, Gosseling T, Heinecke A, Domagk D, Domschke W and Meister T: Clinical outcome and predictors of survival in patients with Pneumocystis jiroveci pneumonia-results of a tertiary referral centre. Clin Respir J 8 : $86-92,2014$

6. Espinel-Ingroff A: Novel antifungal agents, targets or therapeutic strategies for the treatment of invasive fungal diseases: A review of the literature (2005-2009). Rev Iberoam Micol 26: 15-22, 2009

7. Kutty G, Davis AS, Ma L, Taubenberger JK and Kovacs JA: Pneumocystis encodes a functional endo- $\beta-1,3$-glucanase that is expressed exclusively in cysts. J Infect Dis 211: 719-728, 2015.

8. Tu GW, Ju MJ, Xu M, Rong RM, He YZ, Xue ZG, Zhu TY and Luo Z: Combination of caspofungin and low-dose trimethoprim/sulfamethoxazole for the treatment of severe Pneumocystis jirovecii pneumonia in renal transplant recipients. Nephrology (Carlton) 18: 736-742, 2013.

9. Utili R, Durante-Mangoni E, Basilico C, Mattei A, Ragone E and Grossi P: Efficacy of caspofungin addition to trimethoprim-sulfamethoxazole treatment for severe pneumocystis pneumonia in solid organ transplant recipients. Transplantation 84: 685-688, 2007.

10. Lobo ML, Esteves F, de Sousa B, Cardoso F, Cushion MT, Antunes $\mathrm{F}$ and Matos $\mathrm{O}$ : Therapeutic potential of caspofungin combined with trimethoprim-sulfamethoxazole for pneumocystis pneumonia: A pilot study in mice. Plos One 8: e70619, 2013.
11. Mu XD, Que CL, He B, Wang GF and Li HC: Caspofungin in salvage treatment of severe pneumocystis pneumonia: Case report and literature review. Chin Med J (Engl) 122: 996-999, 2009.

12. Beltz K, Kramm CM, Laws HJ, Schroten H, Wessalowski R and Göbel U: Combined trimethoprim and caspofungin treatment for severe Pneumocystis jiroveci pneumonia in a five year old boy with acute lymphoblastic leukemia. Klin Padiatr 218: 177-179, 2006.

13. Kim T, Hong HL, Lee YM, Sung H, Kim SH, Choi SH, Kim YS, Woo JH and Lee SO: Is caspofungin really an effective treatment for Pneumocystis jirovecii pneumonia in immunocompromised patients without human immunodeficiency virus infection? Experiences at a single center and a literature review. Scand J Infect Dis 45: 484-488, 2013.

14. Technical recommendations and guidelines for bronchoalveolar lavage (BAL). Report of the European Society of Pneumology Task Group. Eur Respir J 2: 561-585, 1989.

15. Armstrong-James D, Stebbing J, John L, Murungi A, Bower M, Gazzard B and Nelson M: A trial of caspofungin salvage treatment in PCP pneumonia. Thorax 66: 537-538, 2011.

16. Moon SM, Kim T, Sung H, Kim MN, Kim SH, Choi SH, Jeong JY, Woo JH, Kim YS and Lee SO: Outcomes of moderate-to-severe pneumocystis pneumonia treated with adjunctive steroid in non-HIV-infected patients. Antimicrob Agents Chemother 55: 4613-4618, 2011.

17. Kim T, Kim SH, Park KH, Cho OH, Sung H, Kim MN, Choi SH, Jeong JY, Woo JH, Kim YS and Lee SO: Clindamycin-primaquine versus pentamidine for the second-line treatment of pneumocystis pneumonia. J Infect Chemother 15: 343-346, 2009.

18. Fillatre P, Chevrier S, Revest M, Gacouin A, Jouneau S, Leroy H, Robert-Gangneux F, Minjolle S, Le Tulzo Y and Tattevin P: Human herpes virus co-infection is associated with mortality in HIV-negative patients with Pneumocystis jirovecii pneumonia. Eur J Clin Microbiol Infect Dis 32: 189-194, 2013.

19. Kofteridis DP, Valachis A, Velegraki M, Antoniou M, Christofaki M, Vrentzos GE, Andrianaki AM and Samonis G: Predisposing factors, clinical characteristics and outcome of Pneumonocystis jirovecii pneumonia in HIV-negative patients. J Infect Chemother 20: 412-416, 2014.

20. Linke MJ, Ashbaugh A, Collins MS, Lynch K and Cushion MT: Characterization of a distinct host response profile to Pneumocystis murina asci during clearance of pneumocystis pneumonia. Infect Immun 81: 984-995, 2013.

21. Boonsarngsuk V, Sirilak S and Kiatboonsri S: Acute respiratory failure due to pneumocystis pneumonia: Outcome and prognostic factors. Int J Infect Dis 13: 59-66, 2009.

22. Hardak E, Neuberger A, Yigla M, Berger G, Finkelstein R, Sprecher H and Oren I: Outcome of Pneumocystis jirovecii pneumonia diagnosed by polymerase chain reaction in patients without human immunodeficiency virus infection. Respirology 17: 681-686, 2012.

23. Li MC, Lee NY, Lee CC, Lee HC, Chang CM and Ko WC: Pneumocystis jiroveci pneumonia in immunocompromised patients: Delayed diagnosis and poor outcomes in non-HIV-infected individuals. J Microbiol Immunol Infect 47: 42-47, 2014.

24. Guo F, Chen Y, Yang SL, Xia H, Li XW and Tong ZH: pneumocystis pneumonia in HIV-infected and immunocompromised non-HIV infected patients: A retrospective study of two centers in China. PLoS One 9: e101943, 2014

25. Mu XD, Jia P, Gao L, Su L, Zhang C, Wang RG and Wang GF: Relationship between radiological stages and prognoses of pneumocystis pneumonia in non-AIDS immunocompromised patients. Chin Med J (Engl) 129: 2020-2025, 2016. 